\title{
Application of Nurse Patient Communication in Nursing Care of Elderly Patients with Diabetes Mellitus
}

\author{
Yingzhen Zhao, Tian Tian* \\ Department of Endocrinology, Shaanxi Provincial People’s Hospital, 256 Youyi West Road, Xi’an, China.
}

How to cite this paper: Yingzhen Zhao, Tian Tian. (2021) Application of Nurse Patient Communication in Nursing Care of Elderly Patients with Diabetes Mellitus. International Journal of Clinical and Experimental Medicine Research, 5(3), 284-289.

DOI: 10.26855/ijcemr.2021.07.009

Received: May 13, 2021

Accepted: June 4, 2021

Published: June 9, 2021

*Corresponding author: Tian Tian, Department of Endocrinology, Shaanxi Provincial People's Hospital, 256 Youyi West Road, Xi'an, China.

\begin{abstract}
Objective: To analyze the application of nurse patient communication in nursing care of elderly patients with diabetes mellitus. Methods: 100 elderly patients with diabetes in our hospital from January 2019 to January 2020 were selected retrospectively, and they were divided into routine nursing group and nurse patient communication group (nurse patient communication group) based on routine nursing, with 50 cases in each group. The psychological state, treatment compliance, blood glucose level, quality of life and nursing satisfaction of the two groups were statistically analyzed. Results: After nursing, SAS score and SDS score of nurse patient communication group were lower than those of routine nursing group $(P<0.05)$. The proportion of medication compliance, diet compliance, exercise compliance and life compliance in nurse patient communication group was higher than that in routine nursing group $(P<0.05)$. After nursing, the fasting blood glucose, $2 \mathrm{~h}$ postprandial blood glucose and glycosylated hemoglobin in the nurse patient communication group were lower than those in the routine nursing group $(P<0.05)$. The scores of physiology, vitality, psychology and society in nurse patient communication group were higher than those in routine nursing group $(P<0.05)$. The nursing satisfaction of the nurse patient communication group was higher than that of the routine nursing group $(P<0.05)$. Conclusion: The application effect of nurse patient communication in the nursing of elderly patients with diabetes is good.
\end{abstract}

\section{Keywords}

Old age, diabetes, routine nursing, nurse patient communication, mentality, treatment compliance, blood glucose level, quality of life, nursing satisfaction

Diabetes is a common chronic disease, the main feature of the disease is the chronic increase of blood glucose level, in which type 2 diabetes is common. In the incidence of diabetes, the elderly is the main group, and the elderly group due to poor self-care ability, cognitive obsolescence and combined diseases, which makes diabetes treatment more difficult, so in the elderly diabetes treatment period also need to do a good job of nursing intervention. In the routine nursing for elderly patients with diabetes, nursing staff often focus on the improvement of the condition, often lack of good nurse patient communication, which often makes the patients do not actively cooperate with the treatment, resulting in poor treatment effect, and increase the incidence of nurse patient disputes [1]. In recent years, in our hospital from January 2019 to January 2020 in 100 cases of elderly patients with diabetes, the application of nurse patient communication intervention mode, achieved satisfactory results. 


\section{Materials and methods}

\subsection{General information}

100 elderly patients with diabetes in our hospital from January 2019 to January 2020 were selected retrospectively. According to the nursing methods, they were divided into routine nursing group and nurse patient communication group (nurse patient communication group) on the basis of routine nursing. In the nurse patient communication group, 50 patients were aged from 61 to 82 years old, with an average of $(71.3 \pm 22.5)$ years. There were 21 females (42.00\%) and 29 males (58.00\%). In terms of course distribution, 41 cases (82.00\%) were 3-7 years old and 9 cases $(18.00 \%)$ were 8-15 years old; in terms of complications, 7 cases $(14.00 \%)$ were osteoarthritis, 4 cases $(8.00 \%)$ were hypertension, and 3 cases $(6.00 \%)$ were coronary heart disease. The age of 50 patients in the routine nursing group was $62-83$ years old, with an average of (72.5 \pm 3 3.6). There were 22 females $(44.00 \%)$ and 28 males (56.00\%). In terms of course distribution, 40 cases $(80.00 \%)$ were 3-7 years old and 10 cases $(20.00 \%)$ were $8-15$ years old; In terms of complications, 6 cases $(12.00 \%)$ were osteoarthritis, 5 cases $(10.00 \%)$ were hypertension, and 2 cases $(4.00 \%)$ were coronary heart disease. There was no significant difference in general information between the two groups $(P>0.05)$.

\subsection{Inclusion and exclusion criteria}

Inclusion criteria: (1) all patients met the relevant diagnostic criteria in the expert consensus on diagnosis and treatment of elderly type 2 diabetes in China (2018 Edition); (2) All patients had complete clinical data and no cognitive impairment; (3) Informed consent was signed by patients or their families. The selected cases were approved by the ethics committee of our hospital. Exclusion criteria: (1) patients with severe organic diseases such as heart, liver and kidney; (2) Patients younger than 60 years old; (3) Mental illness or cognitive impairment; (4) Patients who dropped out of the study [2].

\subsection{Methods}

\subsubsection{Routine nursing group}

It mainly includes the importance of regular and quantitative medication to patients on time, maintaining the rationality of diet, formulating a scientific and effective diet plan according to the patient's specific condition, guiding the diet to follow the principles of low sugar, low salt, low fat, high vitamin and light, paying attention to eating less and more meals, avoiding overeating, and avoiding eating animal viscera, egg yolk, greasy and irritating food as far as possible, Emphasize its strict implementation; Guide patients to monitor their blood glucose and body weight, exercise properly, obese people will lose weight regularly, and ask them to come to the hospital regularly.

\subsubsection{Nurse patient communication group}

\subsubsection{Appearance and behavior}

Nurses should keep good appearance, proper clothes, natural appearance and behavior, so as to give patients and their families a good first effect. When communicating with patients, nurses should also face patients with a sincere smile. In the process of communicating with patients with diabetes, a sincere smile is often more effective than gorgeous language, and can eliminate the strangeness between nurses and patients in the process of communication, so that patients can feel the respect of nurses for themselves, so that patients can have full trust in nurses. After the establishment of trust, patients do strictly follow the medical treatment, which can help patients better control their blood glucose levels.

\subsubsection{Reasonable use of language}

In the process of communication between nurses and patients, considering that there are great differences between the elderly patients with diabetes and their cognition of the disease, it is necessary to select different expression methods according to the differences of educational level, gender and age, so as to promote the patients to better understand the meaning that nurses need to express, ensure the communication effect. Nurses should simplify their own language when communicating, and avoid manifesting restlessness, so that patients are more willing to accept it. For example, for patients whose blood glucose control is not ideal, encourage patients to express their usual situation, and then carry out targeted guidance, correct the wrong way of medication, so that the blood glucose level can be effectively controlled.

\subsubsection{Reasonable use of body language}

Nurses should be good at using body language when communicating with patients. For example, when commu- 
nicating with patients, they should look at patients head up. If they have height difference with patients, they can change standing communication to sitting communication, so that nurses and patients can have equal status. If the patients feel sad when they are talking about their experiences, the nursing staff can tap the patient's shoulder and hand over the tissue. The patients with good performance can be encouraged by applause and thumbs up to enhance their self-confidence.

\subsection{Observation index}

\subsubsection{Mental state}

Self-Rating Anxiety Scale (SAS) and Self-Rating Depression Scale (SDS) compiled by Zung were used to evaluate the mental state. SAS was divided into 50 points, 50-59 points was mild anxiety, 60-69 points was moderate anxiety, and more than 69 points was severe anxiety. In China, SDS score $\geq 50$ is regarded as depressive symptoms, and the degree of depression is positively correlated with the scale score [3].

\subsubsection{Treatment compliance}

The treatment compliance of the two groups was investigated, including medication compliance, diet compliance, exercise compliance and life compliance. A questionnaire was set up, with "compliance" and "non-compliance" as the options, and the number and proportion of "compliance" cases were counted.

\subsubsection{Blood glucose level}

Blood glucose meter was used to measure, and the daily average value was taken as the specific value. Blood glucose indexes included fasting blood glucose, $2 \mathrm{~h}$ postprandial blood glucose and glycosylated hemoglobin.

\subsubsection{Quality of life}

SF-36 scale was used to evaluate the quality of life of patients, including physical, psychological, social and vitality, with 100 points for each item, and the score was directly proportional to the quality of life [4].

\subsubsection{Nursing satisfaction}

The patients were asked to fill in the nursing satisfaction questionnaire designed by our hospital. The satisfaction of nursing staff was evaluated from the aspects of nursing staff's service attitude, nursing professional level and patient's comfort, which was divided into three levels: very satisfied, relatively satisfied and dissatisfied. The total satisfaction was the ratio of the sum of satisfaction and the number of general patients.

\subsection{Statistical analysis}

SPSS20.0 statistical software was used for analysis, the measurement data was expressed with $(\bar{x} \pm s)$, the comparison was performed with $t$ test, the repeated measurement data was analyzed with ANOVA, the count data was expressed with rate (\%), the comparison was performed with chi square test, $\mathrm{P}<0.05$ was considered as statistically significant.

\section{Results}

\subsection{Comparison of psychological state between two groups before and after nursing}

The SAS score and SDS score of the two groups were lower than those before nursing $(\mathrm{P}<0.05)$; After nursing, the SAS score and SDS score of the patients in the communication group were lower than that of the normal nursing group $(\mathrm{P}<0.05)$ (see Table 1$)$.

Table 1. Comparison of psychological state of patients before and after nursing (score, $\bar{x} \pm s$ )

\begin{tabular}{ccccc}
\hline Groups & $\mathrm{n}$ & Time & SAS scores & SDS scores \\
\hline Nurse patient communication group & 50 & before nursing & $56.18 \pm 1.53$ & $51.23 \pm 1.17$ \\
& & after nursing & $38.51 \pm 1.47$ & $36.47 \pm 1.26$ \\
Routine nursing group & \multirow{2}{*}{50} & before nursing & $56.20 \pm 1.48$ & $51.30 \pm 1.28$ \\
& & after nursing & $47.24 \pm 1.12$ & $43.56 \pm 1.66$ \\
\hline
\end{tabular}

\subsection{Comparison of treatment compliance between the two groups}

The proportion of medication compliance, diet compliance, exercise compliance and life compliance in nurse patient communication group was higher than that in routine nursing group $(\mathrm{P}<0.05)$ (see Table 2$)$. 
Table 2. Comparison of treatment compliance between the two groups [cases (\%)]

\begin{tabular}{cccccc}
\hline Groups & $\mathrm{n}$ & $\begin{array}{c}\text { medication com- } \\
\text { pliance }\end{array}$ & diet compliance & exercise compliance & life compliance \\
\hline $\begin{array}{c}\text { Nurse patient communication } \\
\text { group }\end{array}$ & 50 & $48(96.00)$ & $46(92.00)$ & $44(88.00)$ & $44(88.00)$ \\
$\begin{array}{c}\text { Routine nursing group } \\
\chi^{2}\end{array}$ & 50 & $42(84.00)$ & $39(78.00)$ & $36(72.00)$ & $34(68.00)$ \\
$P$ & & & & 12.830 \\
\end{tabular}

\subsection{Comparison of blood glucose levels before and after nursing in the two groups}

The fasting blood glucose, $2 \mathrm{~h}$ postprandial blood glucose and glycosylated hemoglobin of the two groups after nursing were lower than those before nursing $(\mathrm{P}<0.05)$; After nursing, the fasting blood glucose, $2 \mathrm{~h}$ postprandial blood glucose and glycosylated hemoglobin in the nurse patient communication group were lower than those in the routine nursing group $(\mathrm{P}<0.05)$ (see Table 3$)$.

Table 3. Comparison of blood glucose levels of two groups before and after nursing ( $\bar{x} \pm s)$

\begin{tabular}{cccccc}
\hline Groups & $\mathrm{n}$ & Time & $\begin{array}{c}\text { fasting blood glucose } \\
(\mathrm{mmol} / \mathrm{L})\end{array}$ & $\begin{array}{c}2 \text { h postprandial blood glu- } \\
\text { cose (mmol/L) }\end{array}$ & $\begin{array}{c}\text { glycosylated hemog- } \\
\text { lobin (\%) }\end{array}$ \\
\hline $\begin{array}{c}\text { Nurse patient communica- } \\
\text { tion group }\end{array}$ & 50 & $\begin{array}{c}\text { before } \\
\text { nursing } \\
\text { after nurs- } \\
\text { ing } \\
\text { before } \\
\text { nursing } \\
\text { after nurs- } \\
\text { ing }\end{array}$ & $8.11 \pm 1.51$ & $10.24 \pm 1.70$ & $7.01 \pm 1.25$ \\
& 50 & $7.23 \pm 1.02$ & $10.13 \pm 1.71$ & $7.21 \pm 0.75$ \\
\end{tabular}

\subsection{Comparison of quality of life between the two groups}

The scores of physiology, vitality, psychology and society in nurse patient communication group were higher than those in routine nursing group $(\mathrm{P}<0.05)$ (see Table 4$)$.

Table 4. Comparison of quality of life between the two groups (points, $\bar{x} \pm s$ )

\begin{tabular}{cccccc}
\hline Groups & $\mathrm{n}$ & physiology & vitality & psychology & society \\
\hline Nurse patient communication group & 50 & $91.57 \pm 6.72$ & $91.67 \pm 6.66$ & $92.10 \pm 6.83$ & $91.06 \pm 6.37$ \\
Routine nursing group & 50 & $82.75 \pm 5.53$ & $82.43 \pm 5.82$ & $82.95 \pm 5.71$ & $81.65 \pm 5.48$ \\
$t$ & & 3.306 & 4.303 & 3.300 & 3.365 \\
$P$ & & $<0.05$ & $<0.05$ & $<0.05$ & $<0.05$ \\
\hline
\end{tabular}

\subsection{Comparison of nursing satisfaction between the two groups}

The nursing satisfaction of the nurse patient communication group was higher than that of the routine nursing group $(\mathrm{P}<0.05)$ (see Table 5).

Table 5. Comparison of nursing satisfaction between the two groups [cases (\%)]

\begin{tabular}{cccccc}
\hline Groups & $\mathrm{n}$ & Very satisfied & Quite satisfied & Dissatisfied & nursing satisfaction \\
\hline Nurse patient communication group & 50 & $41(82.00)$ & $7(14.00)$ & $2(4.00)$ & $48(96.00)$ \\
Routine nursing group & 50 & $26(56.00)$ & $8(16.00)$ & $16(32.00)$ & $34(68.00)$ \\
$\chi^{2}$ & & & & 13.340 \\
$P$ & & & & $<0.05$ \\
\hline
\end{tabular}




\section{Discussion}

Diabetes is a common chronic disease, mainly due to the lack of insulin secretion, glucose metabolism disorders lead to continuous high levels of blood glucose. The disease has a high incidence rate in the elderly population, and because elderly patients often have underlying diseases, this often causes metabolic disorders such as electrolytes, fat and protein, which make patients easily merge multiple complications. At the same time, the elderly patients are lack of awareness of new concepts and good health care awareness, which also leads to the poor treatment of elderly patients with diabetes, which affects the quality of life of patients. Therefore, for elderly patients with diabetes, we need to cooperate with effective nursing intervention during treatment. Diabetes, as a common disease of the contemporary elderly, needs a long process of treatment and nursing. Patients need to maintain healthy and regular living habits while using drugs correctly and reasonably to control the stability of the disease [5]. However, due to the poor resistance of the elderly patients, complications are very easy to occur, which poses a serious threat to the physical and mental health of the elderly patients. In the long process of treatment, patients are prone to have negative emotions. In addition, due to the lack of cognition of diabetes, there are some misunderstandings, resulting in poor compliance behavior and self-management ability, which is not conducive to the effect of clinical treatment and nursing.

In the routine nursing work for elderly diabetes, nurses usually focus on the rationality and effect of medication, which cannot achieve satisfactory intervention effect. Relevant studies have shown that giving patients appropriate psychological nursing, especially nurse patient communication at the same time of treatment, plays a positive role in ensuring patients to actively cooperate with treatment and control blood sugar [6]. Harmonious and friendly nurse patient relationship should be established on the basis of good communication. In this study, nurse patient communication was applied to the nursing of patients in the observation group. By effectively carrying out psychological counseling and health education, the communication with patients was enhanced and appropriate communication skills were applied, which effectively improved the correct cognition of patients with diabetes, so as to improve their physical and mental state. It can enhance the patients' trust and improve their compliance behavior. The results showed that after nursing, the SAS score and SDS score of the nurse patient communication group were lower than those of the routine nursing group $(P<0.05)$. The proportion of medication compliance, diet compliance, exercise compliance and life compliance in nurse patient communication group was higher than that in routine nursing group $(P<0.05)$, indicating that nurse patient communication can effectively guarantee the smooth progress of treatment and nursing.

This study focuses on the effective nursing intervention of communication between patients and nurses in the actual nursing work of the observation group. For the elderly diabetic patients, effective communication between nurses and patients during nursing intervention is helpful to the construction of good relationship between nurses and patients, reducing the incidence of medical disputes and improving patients' satisfaction. Communication is an important means of information exchange and transmission between people. With good communication, we can exchange views and emotions, so that we can trust each other. In the nursing process of elderly diabetes, nurses communicate with the patients or their families, so as to grasp the physical and mental condition of the patients, timely guide the patients to release their negative emotions and enhance the patients' confidence in treatment. In the results of this study, the fasting blood glucose, 2 hours after meal and glycosylated hemoglobin in the communication group were lower than that of the normal nursing group $(P<0.05)$. The scores of physiology, vitality, psychology and society in nurse patient communication group were higher than those in routine nursing group $(P<$ 0.05). The scores of physiology, vitality, psychology and society in nurse patient communication group were higher than those in routine nursing group $(P<0.05)$. The nursing satisfaction of patients in the communication group was higher than that of the conventional nursing group $(P<0.05)$, which indicated that the communication between nurses and patients could effectively reduce blood glucose and improve the quality of life and nursing satisfaction.

In conclusion, the application effect of nurse patient communication in the nursing of elderly patients with diabetes is good, which is worthy of clinical application.

\section{References}

[1] Chen fengjuan, Wang Liyang, Zeng Donggui. (2021). Application of remote family nursing plan in nursing management of pregnant diabetes patients [J]. Qilu Nursing Journal, 2021, 27(3): 55-58.

[2] Cui Jingchen, Zhang Puhong, nadila dolikun, et al. (2021). The application status of mobile interactive platform in diabetes patients' health management [J]. Chinese Journal of Health Management, 2021, 15(1): 98-102. 
[3] Xue Wenjun, niuxiaodan, Wang Yanhong. (2021). A survey of the deviation of the elderly patients with type 2 diabetes mellitus in hospital family transition period [J]. Chinese Journal of Nursing, 2021, 56(2): 225-232.

[4] Zhou Yingfeng, Zhang Mengxing, Li Li, et al. (2020). Investigation of the willingness of patients recommended in the practice guide of clinical nursing for gestational diabetes mellitus [J]. Journal of nurse study, 2020, 35(16): 1523-1526.

[5] Gao Junxiang, Qin Jing. (2020). Nursing care of one type 2 diabetic ketosis patients with insulin allergy [J]. Chinese Journal of nursing, 2020, 55(7): 1081-1083.

[6] Yang Hui, Lin Zhanhui. (2020). The influence of responsibility system holistic nursing on the negative mood and blood glucose control effect of type 2 diabetes mellitus patients [J]. International Journal of nursing, 2020, 39(19): 3608-3611. 